\title{
Uso de serviços odontológicos entre idosos brasileiros
}

\author{
Andréa Maria Eleutério de Barros Lima Martins, ${ }^{1}$ \\ Sandhi Maria Barreto ${ }^{2}$ e Isabela Almeida Pordeus ${ }^{3}$
}

Como citar Martins AMEBL, Barreto SM, Pordeus IA. Uso de serviços odontológicos entre idosos brasileiros. Rev Panam Salud Publica. 2007;22(5):308-16.

RESUMO Objetivo. Investigar a utilização de serviços odontológicos e seus determinantes entre idosos brasileiros.

Método. Foram incluídos os idosos participantes do inquérito de saúde bucal do Ministério da Saúde realizado em 2002 e 2003 que usaram algum serviço odontológico pelo menos uma vez na vida. Dentados e edentados foram comparados e analisados separadamente, utilizando regressão logística múltipla.

Resultados. Dos 5009 participantes, 46,0\% eram dentados e 54,0\% edentados. A prevalência de uso de serviço odontológico no último ano foi 26,6 e 10,4\%, respectivamente. Entre dentados, o uso foi maior entre aqueles com maior escolaridade, que percebiam sua mastigação como péssima/ruim e que relataram sensibilidade dolorosa; e menor entre os que não receberam informações sobre saúde bucal, que percebiam sua saúde bucal como ruim/péssima, que tinham menor renda per capita, que precisavam de prótese e já a usavam, que precisavam de prótese e não a usavam, que tinham problemas periodontais e maior número de dentes extraídos. Entre edentados, o uso foi maior entre aqueles com maior escolaridade e os que relataram sensibilidade dolorosa; e menor entre os idosos identificados como não-brancos e os que não receberam informações sobre saúde bucal.

Conclusão. A saúde bucal dos idosos brasileiros é precária e o uso de serviços odontológicos foi menor entre os que mais necessitavam. Diferentes fatores estiveram associados ao uso entre dentados e edentados. Apenas a escolaridade, o acesso a informações sobre saúde bucal e a dor nos dentes ou gengiva nos últimos 3 meses foram comuns aos dois estratos. São necessários investimentos públicos em saúde bucal para reverter esse quadro.

Palavras-chave Assistência odontológica para idosos, desigualdades em saúde, saúde bucal, Brasil.

1 Universidade Estadual de Montes Claros (UNIMONTES), Curso de Odontologia, e Faculdades Unidas do Norte de Minas (FUNORTE)/ Associação Educativa do Brasil (SOEBRAS), Curso de Odontologia, Minas Gerais, Brasil. Correspondência: Avenida Cula Mangabeira 210, Bairro Santo Expedito, CEP 39401-001, Montes Claros, MG, Brasil. Fone: +55-38-3214. 2672; e-mail: andrea martins@viamoc.com.br

2 Universidade Federal de Minas Gerais (UFMG), Faculdade de Medicina, Departamento de Medicina Preventiva, Belo Horizonte (MG), Brasil.

3 Universidade Federal de Minas Gerais (UFMG), Faculdade de Odontologia, Departamento de Odontopediatria e Ortodontia, Belo Horizonte (MG), Brasil.
As doenças bucais comprometem a qualidade de vida e restringem as atividades cotidianas dos indivíduos (1-3). A preocupação com a saúde bucal dos idosos está crescendo devido ao envelhecimento da população mundial, especialmente nos países desenvolvidos (4). Os danos causados pelas doenças bucais aumentam com a idade e comprometem a qualidade de vida, ocasionando o crescimento da demanda por próteses, geralmente não oferecidas pelos serviços públicos no Brasil (5). Nesse país, o quadro de saúde bucal dos idosos é crítico: em 2003 , apenas $10 \%$ tinham mais de 20 dentes na boca, proporção muito inferior à meta proposta pela Organização Mundial da Saúde (OMS) na qual se preconizava que $50 \%$ da população idosa deveriam apresentar mais de 20 dentes na boca até o ano 2000 (6). 
A saúde bucal, assim como a saúde em geral, está correlacionada ao uso de serviços odontológicos $(1,3)$. $\mathrm{O}$ uso desses serviços com a periodicidade e a freqüência apropriadas contribui para a prevenção de doenças em todas as idades e permite o tratamento precoce dos problemas identificados (7). O edentulismo é uma condição extrema e está, geralmente, associado ao menor uso de serviços odontológicos nos estudos epidemiológicos (8-11). Entretanto, mesmo para os edentados, o uso de serviços odontológicos é importante para avaliar a necessidade de utilização ou substituição de próteses dentárias e para o diagnóstico precoce de lesões potencialmente cancerígenas (12). Dessa forma, o acesso aos serviços odontológicos deve ser garantido não apenas para prevenir o edentulismo, como também para melhorar a qualidade de vida e a saúde bucal dos edentados e dentados.

O modelo teórico de Andersen e Davidson é o mais difundido para analisar os determinantes do uso de serviços odontológicos. Uma versão expandida desse modelo foi utilizada em 1997 para a avaliação do uso de serviços odontológicos nos Estados Unidos (13). Nesse estudo, a raça e a idade foram consideradas como variáveis exógenas, enquanto que as características sociodemográficas e o sistema de atenção à saúde bucal foram considerados como variáveis contextuais. As variáveis relacionadas às características da população foram reunidas em três grupos: disponibilidade de recursos, predisposição ao uso e necessidade de tratamento. Segundo os autores, as características de predisposição e de necessidade de tratamento, além de importantes determinantes do uso, são mutáveis frente às políticas de saúde (14).

Sendo assim, o presente estudo utilizou a versão expandida do modelo de Andersen e Davidson (13) para investigar as características associadas ao uso de serviços odontológicos entre os idosos brasileiros dentados e edentados que participaram do inquérito de saúde bucal realizado pelo Ministério da Saúde em todo o território nacional em 2002 e 2003 (15).

\section{MATERIAIS E MÉTODOS}

O presente trabalho utilizou os dados coletados no inquérito Saúde Bucal do Brasil, anteriormente denominado Projeto SB 2000 e atualmente denominado Projeto SB Brasil (15), realizado pelo Ministério da Saúde, conforme proposta da OMS (16). No projeto, foi avaliada a condição de saúde bucal e coletadas informações sociodemográficas assim como informações sobre o uso de serviços odontológicos e a autopercepção da saúde bucal dos participantes. $\mathrm{O}$ inquérito incluiu 108921 indivíduos residentes em 250 municípios em 2002 e 2003 . A amostra foi desenhada para garantir a representatividade das cinco macrorregiões brasileiras (Norte, Nordeste, CentroOeste, Sudeste e Sul) em duas idades índices (5 e 12 anos) e nos quatro estratos etários (18 a 36 meses, 15 a 19 anos, 35 a 44 anos e 65 a 74 anos) (17). Foram feitas entrevistas e exames nos domicílios, escolas e creches por dentistas treinados e calibrados, de acordo com os critérios propostos pela OMS em 1997 (16) e seguindo os princípios éticos estabelecidos pelo Conselho $\mathrm{Na}$ cional de Saúde (CNS) (6). Os dentistas foram calibrados quanto ao exame das condições bucais a partir do cálculo da concordância Kappa ou da concordância percentual. Os calibradores foram treinados para aferir a concordância intra e interexaminadores. Quando os resultados do teste Kappa ou percentual não eram satisfatórios, os examinadores recebiam um novo treinamento, até que o resultado do teste de concordância atingisse níveis satisfatórios [concordância intra-examinadores: condição da coroa, condição da raiz e necessidade de tratamento, > 97,5\%; índice periodontal comunitário (CPI) e índice de perda de inserção (PIP), $>$ 95,0\%. Concordância interexaminadores: condição da coroa, condição da raiz e necessidade de tratamento, $>$ 90,0\%; CPI e PIP, > 80,0\%]. Maiores informações sobre a metodologia adotada no projeto SB Brasil estão disponíveis em outras publicações (18).

Apesar de, comumente, nos estudos epidemiológicos no Brasil, considerarse como idoso o indivíduo com 60 anos ou mais (19), a OMS propôs, em 1997, investigar o estrato de 65 a 74 anos como representativo da população de idosos em levantamentos epidemiológicos de saúde bucal (16). Essa orientação foi acatada no projeto SB Brasil, de modo a facilitar a comparação com resultados internacionais (15).

A variável dependente foi definida como "uso de serviços odontológicos no último ano" e foi construída pela agregação de duas perguntas:

- já foi ao dentista alguma vez na vida? (sim ou não); e

- há quanto tempo? (nunca foi ao dentista, há menos de 1 ano, de 1 a 2 anos, 3 ou mais).

Todos os que usaram serviços odontológicos pelo menos uma vez na vida foram elegíveis para o presente estudo. A variável foi agrupada em duas categorias: os que usaram esses serviços no último ano e os que não os usaram no último ano.

As variáveis independentes, conforme o modelo de Andersen e Davidson (13), foram reclassificadas em cinco subgrupos: exógenas, contextuais, de predisposição, de disponibilidade de recursos e de necessidade de tratamento. A variável "raça", autodeclarada pelo indivíduo conforme cinco categorias (branco, amarelo, índio, negro ou pardo), foi a única variável exógena e foi categorizada em dois grupos (branco, não branco). As variáveis contextuais incluíram: macrorregiões do Brasil (Sudeste, Sul, CentroOeste, Norte, Nordeste), local de residência (urbano ou rural), escolaridade em anos de estudo $(\leq 4,5$ a $8, \geq 9)$, tipo de serviço odontológico (gratuito ou público/filantrópico, não gratuito ou privado/convênios) e acesso a informações sobre como evitar problemas bucais (sim ou não).

As variáveis de predisposição, que englobaram as características individuais, foram: sexo (feminino ou masculino), autopercepção da saúde bucal (ótima/boa, regular, ruim/péssima), autopercepção da mastigação (ótima/ boa, regular, ruim/péssima), percepção sobre se a saúde bucal afeta o relacionamento social (não afeta ou afeta), 
autopercepção da aparência dos dentes e gengivas (ótima/boa, regular, ruim/ péssima) e autopercepção da influência dos dentes e das gengivas na fala (ótima/boa, regular, ruim/péssima). A renda domiciliar per capita distribuída em tercis (201,00 a 12750,00 reais; 117,00 a 200,00 reais; 0,00 a 116,00 reais) foi considerada como variável de disponibilidade de recursos.

As variáveis de necessidade de tratamento incluíram relato de dor de dentes e/ou gengivas nos últimos 3 meses (não ou sim), a autopercepção da necessidade de tratamento (não ou sim), o uso e a necessidade de confecção ou substituição de prótese entre dentados (não usa e não necessita, usa e não necessita, usa e necessita, não usa e necessita), o uso e a necessidade de confecção ou substituição de prótese entre edentados (usa e não necessita, usa e necessita, não usa e necessita), a alteração de tecido mole (não ou sim), o número de dentes cariados, perdidos e obturados (CPOD) em tercis (0 a 19, 20 a 26, 27 a 32), a necessidade de tratamento periodontal (não ou sim), a necessidade de tratamento por cárie (não ou sim) e o número de dentes extraídos (0 a 15, 16 a 24, 25 a 31). As variáveis CPOD, necessidade de tratamento periodontal, necessidade de tratamento por cárie e número de dentes extraídos foram avaliadas apenas entre dentados por não se aplicarem aos edentados.

Inicialmente, a população idosa brasileira dentada foi comparada à edentada quanto às variáveis propostas por Andersen e Davidson utilizando-se o teste do qui-quadrado $\left(\chi^{2}\right)$ e o valor de $P<0,05$. Posteriormente, em cada estrato, os idosos dentados e edentados que usaram os serviços odontológicos no último ano foram comparados aos que não usaram esses serviços, segundo cada grupo de variáveis de interesse. A significância estatística das diferenças encontradas foi aferida pelo teste do $\chi^{2}$ com nível de significância de $5,0 \%$. A seguir, todos os fatores associados significativamente ao uso, com $P \leq$ 0,20 , foram incluídos no modelo multivariado (20) utilizando regressão logística múltipla, sendo retidos os fatores que permaneceram associados ao nível de $P \leq 0,05$. Cada subgrupo definido pelo modelo de Andersen e Davidson foi analisado separadamente. Ao final, foi construído um modelo único para cada estrato, a partir dos fatores retidos nos modelos multivariados. As magnitudes das associações entre a variável dependente e os fatores de interesse foram estimadas pela razão de chances (odds ratio, OR) com intervalo de confiança de 95\% (IC95\%). A análise foi feita com o programa Statistical Package for the Social Sciences (SPSS) 11.0.

\section{RESULTADOS}

Um total de 5349 idosos (65 a 74 anos) participou do inquérito. Desses, 30 não responderam as perguntas referentes à utilização de serviços odontológicos. Entre os 5319 que responderam, $310(5,8 \%)$ nunca haviam usado serviços odontológicos, 4114 (77,4\%) haviam usado esses serviços há mais de 1 ano e 895 (16,8\%) haviam usado os serviços no último ano. Entre os 5009 idosos incluídos na análise, 2305 (46,0\%) eram dentados e 2704 (54,0\%), edentados. Dos dentados, 614 (26,6\%) haviam usado serviços odontológicos no último ano. Entre os edentados, somente $281(10,4 \%)$ haviam usado os serviços no último ano.

A tabela 1 apresenta a distribuição dos participantes dentados e edentados segundo as características de interesse para o presente trabalho, agrupadas conforme o modelo de Andersen e Davidson. Dentados e edentados só não diferiram significativamente com relação à raça e ao local de residência.

As tabelas 2, 3 e 4 apresentam os resultados das análises univariadas considerando o uso de serviços odontológicos no último ano como variável dependente. Os resultados são apresentados separadamente para dentados e edentados. As variáveis local de residência, autopercepção da influência da fala em função da condição dos dentes e da gengiva, autopercepção da necessidade de tratamento e alterações de tecidos moles não foram estatisticamente associadas ao uso de serviços odontológicos no último ano entre dentados. Já entre edentados, as seguintes variáveis não apresentaram associação estatística com o uso de serviços odontológicos no último ano: local de residência, serviço odontológico, sexo, autopercepção da mastigação, autopercepção da influência dos dentes e das gengivas no relacionamento e na fala, autopercepção da aparência de dentes e gengivas, renda per capita e alteração de tecidos moles.

Os resultados da análise multivariada com os fatores que permaneceram associados ao uso de serviços odontológicos no último ano são apresentados na tabela 5. Entre dentados, a prevalência de uso foi maior entre aqueles com maior escolaridade, que perceberam sua mastigação como regular e péssima ou ruim, e entre os que relataram sensibilidade dolorosa nos dentes ou gengivas nos últimos 3 meses. Já a menor prevalência de uso foi evidenciada entre os que não receberam informações sobre saúde bucal, entre os que percebiam sua saúde bucal como regular e ruim ou péssima, entre os com menor renda per capita, entre os que usavam e necessitavam de próteses, entre os que não usavam e necessitavam de próteses, entre os que apresentavam necessidade de tratamento periodontal e entre aqueles com maior número de dentes extraídos.

Entre os edentados, a análise multivariada mostrou uma maior prevalência de uso entre os idosos com maior escolaridade e entre os que relataram sensibilidade dolorosa nos dentes ou gengivas nos últimos 3 meses. A menor prevalência de uso foi verificada entre os não brancos e os que não receberam informações sobre saúde bucal (tabela 5).

\section{DISCUSSÃO}

O presente trabalho evidenciou que, no Brasil, a prevalência de uso dos serviços odontológicos por idosos no último ano foi muito baixa $(16,8 \%)$. Esse resultado denota uma provável dificuldade de acesso, especialmente considerando que 5,8\% dos idosos relataram nunca ter ido ao dentista. A baixa prevalência de uso explica, em parte, as precárias condições de saúde bucal dos idosos identificadas no inquérito. 
TABELA 1. Distribuição da população idosa dentada e edentada, Brasil, 2002 e $2003^{\text {a }}$

\begin{tabular}{|c|c|c|c|c|c|}
\hline \multirow[b]{2}{*}{ Variáveis } & \multicolumn{2}{|c|}{ Dentado } & \multicolumn{2}{|c|}{ Edentado } & \multirow[b]{2}{*}{$P$} \\
\hline & No. & $\%$ & No. & $\%$ & \\
\hline \multicolumn{6}{|l|}{ Variável exógena } \\
\hline \multicolumn{6}{|l|}{ Raça } \\
\hline Branca & 1106 & 48 & 1360 & 51 & \\
\hline Não branca & 1194 & 52 & 1334 & 49 & 0,091 \\
\hline \multicolumn{6}{|l|}{ Variáveis contextuais } \\
\hline \multicolumn{6}{|l|}{ Macrorregião brasileira } \\
\hline Sudeste & 355 & 15 & 659 & 25 & \\
\hline Sul & 663 & 29 & 675 & 25 & \\
\hline Centro-Oeste & 290 & 13 & 404 & 15 & \\
\hline Norte & 327 & 14 & 361 & 13 & \\
\hline Nordeste & 670 & 29 & 605 & 22 & $<0,001$ \\
\hline \multicolumn{6}{|l|}{ Local de residência } \\
\hline Zona urbana & 2033 & 88 & 2356 & 87 & \\
\hline Zona rural & 272 & 12 & 346 & 13 & 0,281 \\
\hline \multicolumn{6}{|l|}{ Escolaridade(anos) } \\
\hline$\leq 4$ & 1714 & 75 & 2279 & 85 & \\
\hline 5 a 8 & 346 & 15 & 291 & 11 & \\
\hline$\geq 9$ & 217 & 10 & 105 & 4 & $<0,001$ \\
\hline \multicolumn{6}{|l|}{ Serviço odontológico } \\
\hline Gratuito & 1076 & 49 & 1134 & 44 & \\
\hline Não gratuito & 1140 & 51 & 1416 & 56 & 0,005 \\
\hline \multicolumn{6}{|c|}{ Acesso a informações sobre saúde bucal } \\
\hline Sim & 1042 & 45 & 1037 & 38 & \\
\hline Não & 1262 & 55 & 1665 & 62 & $<0,001$ \\
\hline \multicolumn{6}{|l|}{ Variáveis de predisposição } \\
\hline \multicolumn{6}{|l|}{ Sexo } \\
\hline Feminino & 1163 & 51 & 1926 & 71 & \\
\hline Masculino & 1142 & 49 & 778 & 29 & $<0,001$ \\
\hline Autopercepção da saúde buc & & & & & \\
\hline Ótima/boa & 960 & 43 & 1642 & 64 & \\
\hline Regular & 765 & 34 & 653 & 25 & \\
\hline Ruim/péssima & 506 & 23 & 275 & 11 & $<0,001$ \\
\hline Autopercepção da mastigaçã & & & & & \\
\hline Ótima/boa & 1050 & 46 & 1453 & 55 & \\
\hline Regular & 604 & 27 & 601 & 23 & \\
\hline Ruim/péssima & 611 & 27 & 578 & 22 & $<0,001$ \\
\hline Autopercepção do relacionar & & & & & \\
\hline Não afeta & 1405 & 67 & 1816 & 77 & \\
\hline Afeta & 687 & 33 & 534 & 23 & $<0,001$ \\
\hline Autopercepção da aparência & & & & & \\
\hline Ótima/boa & 867 & 39 & 1484 & 61 & \\
\hline Regular & 766 & 35 & 616 & 25 & \\
\hline Ruim/péssima & 572 & 26 & 332 & 14 & $<0,001$ \\
\hline Autopercepção da fala & & & & & \\
\hline Ótima/boa & 1358 & 61 & 1663 & 65 & \\
\hline Regular & 526 & 24 & 553 & 22 & \\
\hline Ruim/péssima & 335 & 15 & 342 & 13 & 0,023 \\
\hline Variáveis de disponibilidade de & & & & & \\
\hline Renda per capita em reais ( $\mathrm{F}$ & & & & & \\
\hline 201 a 12750 & 720 & 32 & 780 & 29 & \\
\hline 100 a 200 & 847 & 36 & 1139 & 42 & \\
\hline 0 a 99 & 720 & 32 & 774 & 29 & $<0,001$ \\
\hline Variáveis de necessidade de tr & & & & & \\
\hline Dor de dentes ou gengivas $n$ & & & & & \\
\hline Não & 1588 & 69 & 2282 & 85 & \\
\hline Sim & 715 & 31 & 418 & 15 & $<0,001$ \\
\hline Autopercepção da necessida & & & & & \\
\hline Não & 620 & 27 & 1690 & 63 & \\
\hline Sim & 1683 & 73 & 1009 & 37 & $<0,001$ \\
\hline Alterações de tecidos moles & & & & & \\
\hline Não & 1976 & 86 & 2201 & 82 & \\
\hline Sim & 312 & 14 & 481 & 18 & $<0,001$ \\
\hline
\end{tabular}

${ }^{\mathrm{a}} \mathrm{A}$ análise incluiu 5009 idosos de 65 a 74 anos (2 305 dentados e 2704 edentados).
A prevalência de uso no presente trabalho foi consideravelmente inferior às prevalências de uso dos serviços odontológicos no ano anterior observadas na década de 1980 nos Estados Unidos $(75,6$ e 58,5\%) $(21,22)$ e na década de 1990 no Canadá $(35,2 \%)$ e Grã-Bretanha $(55,8 \%)(9,23)$. Na maior parte dos estudos, as proporções dos que nunca usaram foram bem menores do que os 5,8\% encontrados na presente investigação: 2,0\% na Finlândia em 1971 (24), 1,0\% na Noruega em 1975 (25), 2,0\% na Flórida, Estados Unidos, na década de 1990 (26), 0,7\% na GrãBretanha em 2002 (23). Já outro estudo, realizado em 1989, nos Estados Unidos, constatou que $5,0 \%$ de idosos nunca haviam usado serviços odontológicos, proporção semelhante à observada na presente investigação (27), enquanto que um estudo realizado no sul da China verificou 10,0\% de não uso de serviços odontológicos na zona urbana e $22,0 \%$ na zona rural (28).

Os resultados mostraram que os dentados e os edentados compartilham duas importantes variáveis contextuais associadas ao uso de serviços odontológicos —escolaridade e informações sobre saúde bucal—, mas mostraram também diferenças importantes entre os dois estratos com relação aos demais conjuntos de variáveis analisados pelo modelo de Davidson e Andersen. Entre os edentados, nenhuma variável de predisposição de uso ou disponibilidade de recursos permaneceu associada ao uso de serviços no último ano. Entre os dentados, entretanto, a renda per capita, a autopercepção da mastigação e da saúde bucal mantiveram-se associadas ao uso de serviços odontológicos. Com relação às variáveis de necessidade de tratamento, apenas o relato de dor em dentes ou gengivas foi associada ao uso nos dois estratos. As especificidades observadas em cada estrato analisado são importantes para orientar políticas públicas voltadas para a prevenção do edentulismo, a melhoria da condição de saúde bucal e a redução de desigualdades no uso de serviços odontológicos entre os idosos.

As consultas ao dentista, diferentemente do que ocorre com as visitas ao 
TABELA 2. Análise univariada do uso de serviços odontológicos no último ano pelos idosos segundo raça e variáveis contextuais, Brasil, 2002 e $2003^{\mathrm{a}}$

\begin{tabular}{|c|c|c|c|c|c|c|c|c|}
\hline \multirow[b]{3}{*}{ Variáveis } & \multicolumn{4}{|c|}{ Dentados } & \multicolumn{4}{|c|}{ Edentados } \\
\hline & \multicolumn{2}{|c|}{ Usou } & \multirow[b]{2}{*}{$\mathrm{OR}^{\mathrm{b}}$} & \multirow[b]{2}{*}{ IC $95 \%{ }^{b}$} & \multicolumn{2}{|c|}{ Usou } & \multirow[b]{2}{*}{ OR } & \multirow[b]{2}{*}{ IC95\% } \\
\hline & $n$ & $\%$ & & & $n$ & $\%$ & & \\
\hline \multicolumn{9}{|l|}{ Variável exógena } \\
\hline \multicolumn{9}{|l|}{ Raça } \\
\hline Branca & 355 & 58 & 1,00 & & 167 & 60 & 1,00 & \\
\hline Não branca & 258 & 42 & 0,58 & 0,48 a 0,70 & 112 & 40 & 0,65 & 0,50 a 0,84 \\
\hline \multicolumn{9}{|l|}{ Variáveis contextuais } \\
\hline \multicolumn{9}{|l|}{ Macrorregião brasileira } \\
\hline Sul & 227 & 37 & 1,13 & 0,85 a 1,48 & 95 & 34 & 1,27 & 0,92 a 1,76 \\
\hline Centro-Oeste & 77 & 13 & 0,78 & 0,55 a 1,10 & 31 & 11 & 0,64 & 0,41 a 1,00 \\
\hline Norte & 67 & 11 & 0,55 & 0,39 a 0,79 & 34 & 12 & 0,81 & 0,52 a 1,24 \\
\hline Nordeste & 131 & 21 & 0,52 & 0,39 a 0,70 & 46 & 16 & 0,64 & 0,43 a 0,94 \\
\hline \multicolumn{9}{|l|}{ Local de residência } \\
\hline Zona urbana & 553 & 90 & 1,00 & & 251 & 89 & 1,00 & \\
\hline Zona rural & 61 & 10 & 0,77 & 0,57 a 1,04 & 30 & 11 & 0,79 & 0,53 a 1,18 \\
\hline \multicolumn{9}{|l|}{ Serviço odontológico } \\
\hline Gratuito & 259 & 43 & 1,00 & & 125 & 47 & 1,00 & \\
\hline Não gratuito & 345 & 57 & 1,37 & 1,13 a 1,65 & 142 & 53 & 0,90 & 0,69 a 1,16 \\
\hline \multicolumn{9}{|c|}{ Acesso a informações sobre saúde bucal } \\
\hline Sim & 389 & 63 & 1,00 & & 160 & 57 & 1,00 & \\
\hline Não & 225 & 37 & 0,36 & 0,30 a 0,44 & 121 & 43 & 0,43 & 0,33 a 0,55 \\
\hline
\end{tabular}

${ }^{a} A$ análise incluiu 5009 idosos de 65 a 74 anos (2 305 dentados e 2704 edentados).

${ }^{\mathrm{b}} \mathrm{OR}=$ razão de produtos cruzados (odds ratio); IC95\% = intervalo de confiança de $95 \%$.

TABELA 3. Análise univariada do uso de serviços odontológicos no último ano pelos idosos segundo variáveis de predisposição ao uso e disponibilidade de recursos, Brasil, 2002 e 2003

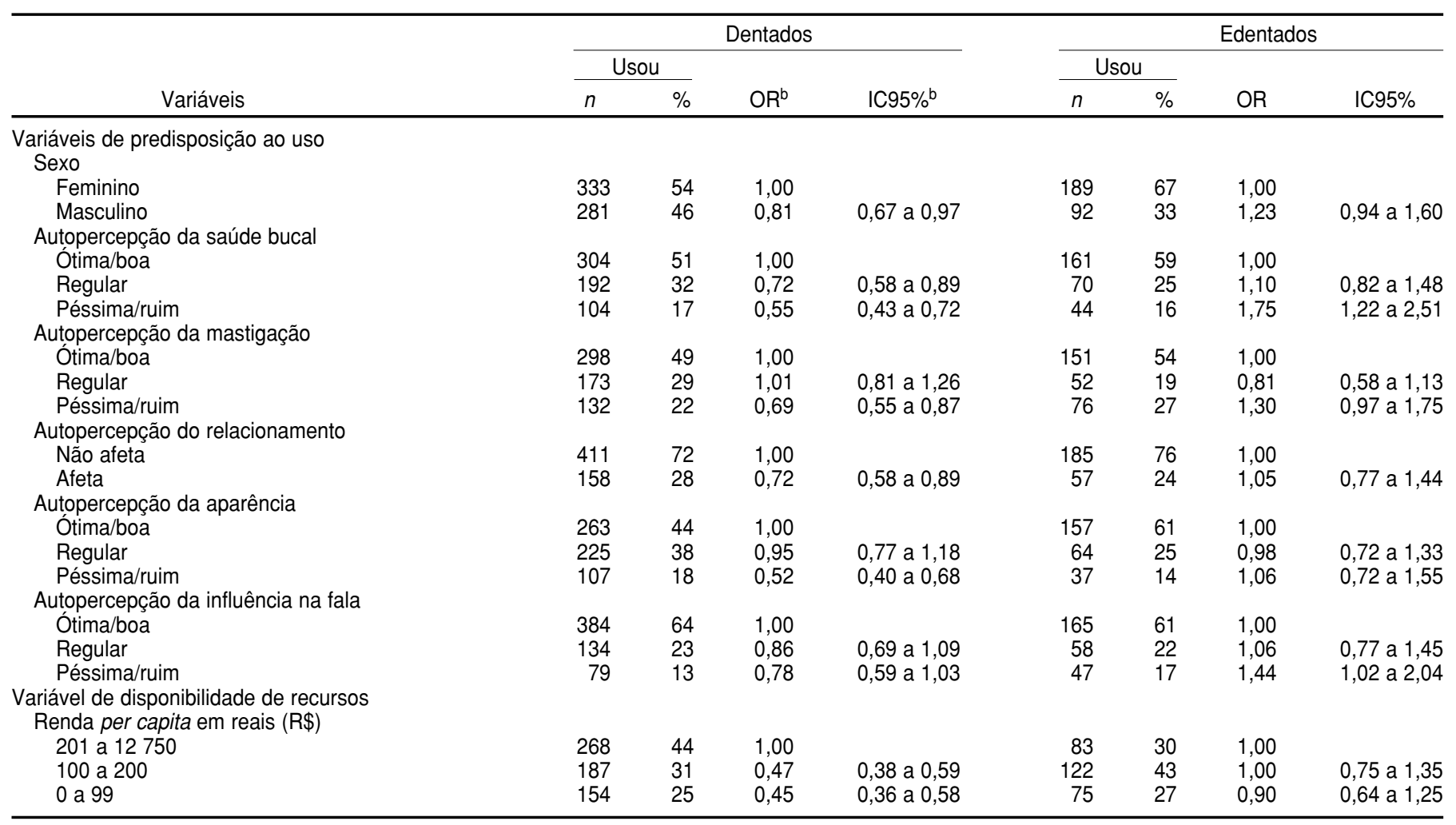

${ }^{\mathrm{a}} \mathrm{A}$ análise incluiu 5009 idosos de 65 a 74 anos (2 305 dentados e 2704 edentados).

${ }^{\mathrm{b}} \mathrm{OR}=$ razão de produtos cruzados (odds ratio); IC $95 \%$ = intervalo de confiança de $95 \%$. 
TABELA 4. Análise univariada do uso de serviços odontológicos no último ano entre os idosos segundo variáveis de necessidade, Brasil, 2002 e $2003^{\mathrm{a}}$

\begin{tabular}{|c|c|c|c|c|c|c|c|c|}
\hline \multirow[b]{3}{*}{ Variáveis de necessidade } & \multicolumn{4}{|c|}{ Dentados } & \multicolumn{4}{|c|}{ Edentados } \\
\hline & \multicolumn{2}{|c|}{ Usou } & \multirow[b]{2}{*}{$\mathrm{OR}^{\mathrm{b}}$} & \multirow[b]{2}{*}{ IC95\% ${ }^{\mathrm{b}}$} & \multicolumn{2}{|c|}{ Usou } & \multirow[b]{2}{*}{ OR } & \multirow[b]{2}{*}{ IC95\% } \\
\hline & $n$ & $\%$ & & & $n$ & $\%$ & & \\
\hline Não & 373 & 61 & 1,00 & & 186 & 66 & 1,00 & \\
\hline Sim & 240 & 39 & 1,64 & 1,35 a 1,99 & 94 & 34 & 3,26 & 2,48 a 4,30 \\
\hline \multicolumn{9}{|c|}{ Autopercepção da necessidade de tratamento } \\
\hline Nã̃o & 174 & 28 & 1,00 & & 149 & 53 & 1,00 & \\
\hline Não necessita & 188 & 30 & 1,00 & & 199 & 71 & 1,00 & \\
\hline Usa e necessita & 212 & 35 & 0,42 & 0,32 a 0,54 & 52 & 19 & 0,97 & 0,70 a 1,35 \\
\hline Não usa e necessita & 213 & 35 & 0,32 & 0,25 a 0,41 & 29 & 10 & 0,57 & 0,37 a 0,85 \\
\hline \multicolumn{9}{|l|}{ Alteração de tecidos moles } \\
\hline Não & 530 & 87 & 1,00 & & 238 & 85 & 1,00 & \\
\hline Sim & 78 & 13 & 0,90 & 0,69 a 1,19 & 41 & 15 & 0,76 & 0,54 a 1,08 \\
\hline \multicolumn{9}{|l|}{$\mathrm{CPOD}^{\mathrm{c}, \mathrm{d}}$} \\
\hline Sim & 383 & 70 & 0,44 & 0,35 a 0,56 & & & & \\
\hline \multicolumn{9}{|l|}{ Necessidade de tratamento por cárie ${ }^{c}$} \\
\hline Não & 7 & 1 & 1,00 & & & & & \\
\hline Sim & 607 & 99 & 4,76 & 2,19 a 10,33 & & & & \\
\hline \multicolumn{9}{|l|}{ Número de dentes extraídos ${ }^{c}$} \\
\hline 0 a 15 & 237 & 39 & 1,00 & & & & & \\
\hline 16 a 24 & 223 & 36 & 0,74 & 0,60 a 0,93 & & & & \\
\hline 25 a 31 & 154 & 25 & 0,50 & 0,39 a 0,63 & & & & \\
\hline
\end{tabular}

${ }^{a}$ A análise incluiu 5009 idosos de 65 a 74 anos (2 305 dentados e 2704 edentados).

b $\mathrm{OR}=$ razão de produtos cruzados (odds ratio); IC95\% = intervalo de confiança de $95 \%$.

${ }^{c}$ Variaveis não avaliadas entre edentados.

${ }^{\mathrm{d}}$ Dentes cariados, perdidos e obturados.

médico, parecem se tornar menos freqüentes com o envelhecimento (4), sendo esperada uma baixa prevalência de uso de serviços odontológicos entre os idosos quando comparados a populações mais jovens $(7,12,29)$. O menor uso parece ser explicado, em grande parte, pela alta freqüência de idosos edentados, uma vez que este fator está associado à menor prevalência de uso de serviços odontológicos em vários estudos $(10,11,29)$. Há indícios de que os edentados acreditam que a visita regular ao dentista é importante apenas para quem ainda tem dentes (30). No presente estudo, a prevalência de uso de serviços odontológicos foi cerca de 3 vezes maior entre os idosos dentados quando comparados aos endentados, sendo evidente a necessidade de conhecer as crenças dos idosos edentados sobre esse aspecto.

A associação inversa entre a escolaridade e o uso de serviços, observada nos dois estratos, já foi verificada em estudos conduzidos no Brasil (12) e em outros países (29-31). O menor uso entre os que não tiveram acesso a informações sobre saúde bucal é um resultado interessante e sugere a necessidade de difundir as informações relacionadas à saúde bucal entre os idosos. Resultados semelhantes também foram encontrados na Dinamarca e nos Estados Unidos $(21,31)$. Chama atenção e merece investigação o menor uso de serviços pelos edentados identificados como não brancos.

A influência da renda no acesso à consulta odontológica tende a ser maior nos países em que esses serviços são predominantemente pagos. No Brasil, $44 \%$ dos idosos utilizaram serviços odontológicos gratuitos, o que reflete um acesso ainda limitado ao serviço odontológico público. Verificou-se uma relação direta entre a renda e o uso de serviços em inquéritos feitos nos Estados Unidos (21), Canadá (9-11) e Dinamarca (31). O fato de a renda não ter aparecido como variável associada ao uso no último ano entre os edentados pode ser devido à homogeneidade de renda nesse estrato, que concentra uma população pobre, ou mesmo a um problema de acesso desse estrato aos serviços gratuitos.

A dor em dentes ou na gengiva nos últimos 3 meses foi a única variável de necessidade associada ao uso dos serviços odontológicos no último ano comum aos dois estratos. Essa associação também foi encontrada por Davidson e Andersen (29) e sugere que a procura por assistência odontológica se dá quando a saúde bucal declina consideravelmente (4). Entre os edentados, não é possível afirmar, mas não se pode descartar, a possibilidade de essa associação indicar que o uso teve como finalidade a realização de extração dos dentes remanescentes. 
TABELA 5. Características associadas ao uso de serviços odontológicos pelos idosos na análise multivariada, Brasil, 2003a

\begin{tabular}{|c|c|c|}
\hline & Dentados & Edentados \\
\hline Variáveis & OR $(\text { IC95\%) })^{b}$ & OR (IC95\%) \\
\hline \multicolumn{3}{|l|}{ Variável exógena } \\
\hline \multicolumn{3}{|l|}{ Raça } \\
\hline Branca & 1,00 & 1,00 \\
\hline Não branca & NSD & $0,74(0,56$ a 0,96$)$ \\
\hline \multicolumn{3}{|l|}{ Variáveis contextuais } \\
\hline \multicolumn{3}{|c|}{ Escolaridade (anos de estudo) } \\
\hline$\leq 4$ & 1,00 & 1,00 \\
\hline 5 a 8 & $1,45(1,07$ a 1,97$)$ & $1,63(1,14$ a 2,34$)$ \\
\hline$\geq 9$ & $2,04(1,43$ a 2,90$)$ & $2,98(1,84$ a 4,83$)$ \\
\hline \multicolumn{3}{|c|}{ Acesso a informações sobre saúde bucal } \\
\hline Sim & 1,00 & 1,00 \\
\hline Não & $0,48(0,38$ a 0,60$)$ & $0,46(0,35$ a 0,60$)$ \\
\hline \multicolumn{3}{|c|}{ Variáveis de predisposição ao uso } \\
\hline \multicolumn{3}{|c|}{ Autopercepção da saúde bucal } \\
\hline Ótima/boa & 1,00 & 1,00 \\
\hline Regular & $0,59(0,45$ a 0,78$)$ & $\mathrm{NSE}^{\mathrm{d}}$ \\
\hline Péssima/ruim & $0,45(0,31$ a 0,64$)$ & $\mathrm{NSE}^{\mathrm{d}}$ \\
\hline \multicolumn{3}{|c|}{ Autopercepção da mastigação } \\
\hline Ótima/boa & 1,00 & 1,00 \\
\hline Regular & $1,36(1,02$ a 1,81$)$ & NSE $^{d}$ \\
\hline Péssima/ruim & $1,42(1,01$ a 2,00$)$ & NSE $^{d}$ \\
\hline \multicolumn{3}{|c|}{ Variáveis de disponibilidade de recursos } \\
\hline \multicolumn{3}{|c|}{ Renda per capita em reais $(\mathrm{R} \$)$} \\
\hline 201 a 12750 & 1,00 & 1,00 \\
\hline 100 a 200 & $0,65(0,50$ a 0,86$)$ & NSE $^{d}$ \\
\hline 0 a 99 & $0,58(0,43$ a 0,77$)$ & NSE $^{d}$ \\
\hline \multicolumn{3}{|l|}{ Variáveis de necessidade } \\
\hline \multicolumn{3}{|c|}{ Dor em dentes ou gengivas nos últimos 3 meses } \\
\hline Não & 1,00 & 1,00 \\
\hline $\operatorname{Sim}$ & $1,98(1,54$ a 2,54$)$ & $3,24(2,44$ a 4,30$)$ \\
\hline \multicolumn{3}{|l|}{ Prótese } \\
\hline Não necessita & 1,00 & 1,00 \\
\hline Usa e necessita & $0,60(0,44$ a 0,81$)$ & NSEd \\
\hline Não usa e necessita & $0,48(0,34$ a 0,66$)$ & $\mathrm{NSE}^{\mathrm{d}}$ \\
\hline \multicolumn{3}{|l|}{ Tratamento periodontale } \\
\hline Não & 1,00 & 1,00 \\
\hline Sim & $0,57(0,44$ a 0,75$)$ & - \\
\hline \multicolumn{3}{|l|}{ Número de dentes extraídos ${ }^{\mathrm{e}}$} \\
\hline 0 a 15 & 1,00 & 1,00 \\
\hline 16 a 24 & $0,71(0,54$ a 0,94$)$ & - \\
\hline
\end{tabular}

${ }^{a}$ A análise incluiu 5009 idosos de 65 a 74 anos (2 305 dentados e 2704 edentados).

${ }^{b} \mathrm{OR}=$ razão de produtos cruzados (odds ratio); 1 IC95\% = intervalo de confiança de $95 \%$.

${ }^{\mathrm{C}} \mathrm{NSD}=$ Não significativamente associado entre dentados.

${ }^{d}$ NSE = Não significativamente associado entre dentados.

e Variáveis não avaliadas entre edentados.

O maior uso de serviços entre os idosos dentados que perceberam a sua mastigação como regular e ruim/ péssima sugere que a limitação ou deficiência mastigatória prediz o uso de serviços entre os dentados. $\mathrm{O}$ fato de não aparecer como variável relacionada ao uso entre edentados pode indicar acomodação a uma pior qualidade de mastigação ou deficiência de acesso aos serviços nesse estrato. É surpreendente a menor freqüência de uso entre os dentados que autopercebiam a sua saúde bucal como regular ou péssima/ruim. Problemas de acesso a serviços odontológicos, assim como uma certa resignação com a condição de saúde bucal não podem ser descartados.

Nos dois estratos analisados no presente trabalho, diferentemente de outros estudos, não foram verificadas as- sociações entre o uso de serviços odontológicos no último ano e o sexo (29), a macrorregião de residência ou a residência em zona urbana e rural (11, 12, 31). A autopercepção da aparência, da fala e do relacionamento social em função das condições bucais não estiveram associadas ao uso de serviços odontológicos, indicando a necessidade de avaliar melhor o papel dessas variáveis no cuidado com os dentes.

O uso e a necessidade de prótese não estiveram associados ao uso de serviços odontológicos entre os edentados no presente estudo. A literatura mostra resultados inconsistentes sobre o tema $(21,29,31)$. É possível que os edentados brasileiros não tenham informação satisfatória sobre a necessidade de consultas regulares ao dentista para avaliação e manutenção de suas próteses. A menor prevalência de uso entre os idosos dentados que necessitam de prótese ou de tratamento periodontal, assim como a associação inversa com o número de dentes extraídos e a forte associação entre as visitas ao dentista e a dor —uma expressão da necessidade de tratamento- podem retratar a iniqüidade (pois os que mais necessitam são os que menos usam) ou uma tendência à procura pelo dentista somente em situações de urgência.

Tais resultados preocupam, pois a piora na saúde bucal tende a levar ao edentulismo se não for tratada a tempo e adequadamente. Chama atenção o fato de a maioria dos idosos dentados no presente estudo estar próxima da condição de edentulismo pela precariedade da sua condição bucal e pelo pequeno número de dentes remanescentes na boca. Um fator que pode concorrer para explicar a associação negativa entre a necessidade de prótese e o uso de serviços é o custo elevado do tratamento protético. Vale salientar que a relação entre o uso de serviços odontológicos e as variáveis investigadas é dinâmica, portanto causas e efeitos certamente variam ao longo da vida. Sendo este um estudo seccional, não é possível estabelecer uma relação temporal entre as associações observadas. Quem menos usa os serviços para tratamento restaurador e preventivo 
tende a perder mais dentes e ter uma pior condição geral de saúde bucal em decorrência do menor uso.

Dentados e edentados mostraram diferenças importante com relação às variáveis associadas ao uso de serviços odontológicos. Apenas a escolaridade, o relato de ter recebido informações sobre saúde bucal e o relato de dor de dente ou gengiva nos últimos 3 meses foram variáveis comuns aos dois estratos. Este trabalho também evidenciou um menor uso de serviços entre os idosos com saúde bucal mais precária, ou seja, entre os que mais necessitavam de tratamento, o que sugere iniqüidade no acesso aos serviços odontológicos. A menor prevalência de uso entre aqueles que não receberam informações sobre saúde bucal sugere a necessidade de melhorar a qualidade dos serviços preventivos, que devem passar a oferecer orientações sobre o autoexame bucal para a identificação precoce dos problemas bucais.

A necessidade de tratamento e de revisão das próteses e a suspeita da neoplasia são razões para a procura dos serviços odontológicos. No presente estudo, importantes variáveis de necessidade de tratamento dentário estiveram associadas ao menor uso de serviços, o que pode levar a um ciclo vicioso de deterioração da saúde bucal. A população idosa está crescendo e necessita de políticas de saúde bucal específicas para reduzir o edentulismo e melhorar as condições gerais de saúde e de vida. Para tanto, são necessários investimentos em saúde bucal que possam garantir o acesso e aumentar a motivação para o uso de serviços odonto- lógicos preventivos e regulares, tanto entre os dentados quanto entre os edentados, revertendo, assim, o precário quadro de saúde bucal e reduzindo as desigualdades observadas.

Agradecimentos. Agradecemos à Coordenação de Saúde Bucal do Ministério da Saúde e à equipe de campo do inquérito, bem como aos participantes do estudo. Andréa Maria Eleutério de Barros Lima Martins recebeu bolsa das Faculdades Unidas de Norte de Minas (FUNORTE)/Associação Educativa do Brasil (SOEBRAS) durante a realização deste trabalho. Sandhi Maria Barreto e Isabela Almeida Pordeus são bolsistas de produtividade em pesquisa do Conselho Nacional de Desenvolvimento Científico e Tecnológico (CNPq).

\section{REFERÊNCIAS}

1. Petersen PE. The Word Oral Health Report 2003. Continuous improvement of oral health in the 21st century-the approach of the WHO Global Oral Health Programme. Community Dent Oral Epidemiol. 2003;31(1):3-24.

2. Reisine ST. Dental disease and work loss. J Dent Res. 1984;63(9): 1158-61.

3. Reisine ST. The impact of dental conditions on social functioning and the quality of life. Annual Rev Public Health. 1988;9:1-19.

4. Kiyak HA. Explaining patterns of dental service utilization among the elderly. J Dent Educ. 1986;50(11):679-87.

5. Colussi CF, Freitas SFT. Aspectos epidemiológicos da saúde bucal do idoso no Brasil. Cad Saude Publica. 2002;18(5):1313-20.

6. Brasil, Ministério da Saúde, Coordenação Nacional de Saúde Bucal. Resultados principais do projeto SB Brasil 2003: condições de saúde bucal da população brasileira 2002-2003. Brasília: Ministério da Saúde; 2004

7. Newman JF, Gift HC. Regular pattern of preventive dental services-a measure of access. Soc Sci Med. 1992;35(38):997-1001.

8. Gilbert GH, Branch LG, Longmate J. Dental care use by U.S. veterans eligible for VA Care. Soc Sci Med. 1993;36(3):361-70.

9. Locker D, Ford J. Evaluation of an area-based measure as an indicator of inequalities in oral health. Community Dent Oral Epidemiol. 1994;22(2):80-5.

10. Locker D, Ford J. Using area-based measures of socioeconomic status in dental health ser- vices research. J Public Health Dent. 1996;56 (2):69-75.

11. Locker D, Leake JL, Lee J, Main PA, Hicks T, Hamilton M. Utilization of dental services by older adults in four Ontario communities. J Can Dent Assoc. 1991;57(11):879-86.

12. Matos DL, Giatt, L, Lima Costa MF. Fatores sócio-demográficos associados ao uso de serviços odontológicos entre idosos brasileiros: um estudo baseado na Pesquisa Nacional por amostras de domicílio. Cad Saude Publica. 2004;20(5):1290-7.

13. Andersen RM, Davidson PL. Ethnicity, aging, and oral health outcomes: a conceptual framework. Adv Dent Res. 1997;11(2):203-9.

14. Gift HC, Atchison KA, Drury TF. Perceptions of the natural dentition in the context of multiple variables. J Dent Res. 1998;77(7): 1529-38.

15. Brasil, Ministério da Saúde. Projeto SB 2000: Condições da saúde bucal da população brasileira, 2000. Brasília: Ministério da Saúde; 2000.

16. World Health Organization. Oral health surveys: basic methods. 4th ed. Genebra: WHO; 1997.

17. Brasil, Ministério da Saúde, Secretaria de Atenção à Saúde. Projeto SB 2000: condições de saúde bucal da população brasileira no ano 2000. Manual do coordenador. Brasília: Ministério da Saúde; 2001.

18. Brasil, Ministério da Saúde, Secretaria de Atenção à Saúde. Projeto SB 2000: condições de saúde bucal da população brasileira no ano
2000. Manual de calibração de examinadores. Brasília: Ministério da Saúde; 2001.

19. Lima-Costa MF, Barreto SM. Tipos de estudos epidemiológicos: conceitos básicos e aplicações na área do envelhecimento. Epidemiol Serv Saude. 2003;12(4):189-201.

20. Greenland S. Modeling and variable selection in epidemiologic analysis. Am J Public Health. 1989;79(3):340-9.

21. Atchison KA, Mayer-Oakes SA, Schweitzer SO, Lubben JE, De Jong FJ, Mathias RE. The relationship between dental utilization and preventive participation among a well-elderly sample. J Publ Health Dent. 1993;53(2):88-95.

22. Ettinger RL. The unique oral health needs of an aging population. Dent Clin North America. 1997;41(4):633-49.

23. Mc Grath C, Bedi R. Influences of social support on the oral health of older people in Britain. J Rehab. 2002;29:918-22.

24. Murtoma AH, Markulla J. Utilization and hindrances to utilization of dental services in Finland prior to the public health. Proc Finn Dent Soc. 1977;73(3):138-43.

25. Heloe LA, Trostand L. The use of dental services among Norwegian adults in 1973. Community Dent Oral Epidemiol. 1975;3(3):120-5.

26. Gilbert GH, Heft MN, Duncan RP, Ringelberg ML. Perceived need for dental care in dentate older adults. Int Dent J. 1994;44(2):145-52.

27. Bolden AD, Joseph L, Henry JL, Allukian M. Implications of access, utilization and need for oral health care by low income groups 
and minorities on the dental delivery system. J Dent Educ. 1993;57(12):888-98.

28. Lo EC, Lin HC, Wang ZJ, Wong MC, Schwarz E. Utilization of dental services in Southern China. J Dent Res. 2001;80(5):1471-4.

29. Davidson PL, Andersen RM. Determinants of dental care utilization for diverse ethnic and age groups. Adv Dent Res. 1997;11(2): 254-62.

30. Tennstedt SL, Brambilla DL, Jette AM. Understanding dental service use by older adults: sociobehavioral factors vs. need. J Public Health Dent. 1994;54(4):211-9.
31. Schwarz E. Changes in demand for dental care among Danish adults, 1975-90. Acta Odontol Scand. 1996;54(1):36-43.

Manuscrito recebido em 16 de março de 2007. Aceito em versão revisada em 1 de agosto de 2007.

ABSTRACT Objective. To investigate the pattern of dental services use and its determinants among the elderly in Brazil.

Methods. The study included the elderly participating in the Ministry of Health of

Utilization of dental services among the elderly in Brazil
Brazil's oral health survey carried out in 2002 and 2003 who had used dental services at least once in their lifetime. Dentulous and edentulous individuals were compared and analyzed separately using multiple logistic regression.

Results. Of 5009 participants, $46.0 \%$ were dentulous and $54.0 \%$ edentulous. The prevalence of dental services use in the year right before the survey was $26.6 \%$ and $10.4 \%$, respectively. Among dentulous participants, the utilization rate was higher in those with more years of schooling, those who perceived their chewing as poor/very poor, and those who reported feeling oral pain; and lower in those who did not have information on oral health, who perceived their oral health as poor/very poor, those with lower per capita income, those who required prostheses and used them, and who required prostheses and did not use them, those with periodontal problems, and with a higher number of extracted teeth. Among edentulous participants, the utilization rate was higher in those with more years of schooling and those reporting oral pain; and lower in non-whites and in those who did not have information on oral health.

Conclusions. The oral health of the elderly in Brazil is deficient and the use of dental services was lower among those who needed them most. The factors associated with dental services utilization differ for dentulous and edentulous individuals. The only similarities are schooling, having information on oral health, and having experienced pain in the teeth and gums in the previous three months. Public investment in oral health is needed to turn around this scenario.

Key words Dental care for aged, health inequalities, oral health, Brazil. 